OPEN ACCESS

Edited by:

Jose M. Gonzalez,

University of La Laguna, Spain

Reviewed by:

Christopher R. Reisch

University of Florida, United States

Helen F. Fredricks,

Woods Hole Oceanographic

Institution, United States

${ }^{*}$ Correspondence:

Yin Chen

Y.chen.25@warwick.ac.uk

Specialty section:

This article was submitted to

Aquatic Microbiology,

a section of the journal

Frontiers in Microbiology

Received: 15 April 2020 Accepted: 25 November 2020 Published: 21 December 2020

Citation:

Silvano $E$, Yang $M$, Wolterink $M$, Giebel H-A, Simon M, Scanlan DJ,

Zhao Y and Chen Y (2020) Lipidomic

Analysis of Roseobacters of the

Pelagic RCA Cluster and Their

Response to Phosphorus Limitation.

Front. Microbiol. 11:552135.

doi: 10.3389/fmicb.2020.552135

\section{Lipidomic Analysis of Roseobacters of the Pelagic RCA Cluster and Their Response to Phosphorus Limitation}

\author{
Eleonora Silvano', Mingyu Yang², Mathias Wolterink ${ }^{3}$, Helge-Ansgar Giebel ${ }^{3}$, \\ Meinhard Simon ${ }^{3}$, David J. Scanlan', Yanlin Zhao ${ }^{2}$ and Yin Chen ${ }^{1 *}$
}

${ }^{1}$ School of Life Sciences, University of Warwick, Coventry, United Kingdom, ${ }^{2}$ Fujian Provincial Key Laboratory of Agroecological Processing and Safety Monitoring, College of Life Sciences, Fujian Agriculture and Forestry University, Fuzhou, China, ${ }^{3}$ Institute for Chemistry and Biology of the Marine Environment (ICBM), University of Oldenburg, Oldenburg, Germany

The marine roseobacter-clade affiliated cluster $(R C A)$ represents one of the most abundant groups of bacterioplankton in the global oceans, particularly in temperate and sub-polar regions. They play a key role in the biogeochemical cycling of various elements and are important players in oceanic climate-active trace gas metabolism. In contrast to copiotrophic roseobacter counterparts such as Ruegeria pomeroyi DSS-3 and Phaeobacter sp. MED193, RCA bacteria are truly pelagic and have smaller genomes. We have previously shown that RCA bacteria do not appear to encode the PlcP-mediated lipid remodeling pathway, whereby marine heterotrophic bacteria remodel their membrane lipid composition in response to phosphorus $(P)$ stress by substituting membrane glycerophospholipids with alternative glycolipids or betaine lipids. In this study, we report lipidomic analysis of six RCA isolates. In addition to the commonly found glycerophospholipids such as phosphatidylglycerol (PG) and phosphatidylethanolamine (PE), RCA bacteria synthesize a relatively uncommon phospholipid, acylphosphatidylglycerol, which is not found in copiotrophic roseobacters. Instead, like the abundant SAR11 clade, RCA bacteria upregulate ornithine lipid biosynthesis in response to $\mathrm{P}$ stress, suggesting a key role of this aminolipid in the adaptation of marine heterotrophs to oceanic nutrient limitation.

Keywords: roseobacter clade bacteria, Ruegeria pomeroyi DSS-3, lipids, marine heterotrophic bacteria, lipid remodeling

\section{INTRODUCTION}

The marine roseobacter group of Alphaproteobacteria comprises an ecologically important group of marine bacteria involved in the biogeochemical cycling of carbon, nitrogen, and sulfur (Buchan et al., 2014; Luo and Moran, 2014). Roseobacters are metabolically diverse, being able to use an array of organic molecules, perform anoxygenic photosynthesis and produce secondary metabolites (Brinkhoff et al., 2008; Buchan et al., 2014). Arguably however, they are most well known for their role in marine trace gas formation, including the metabolism of methylated sulfur compounds like dimethylsulfide and dimethylsulfoniopropionate, and methylated amines, which has global significance (Curson et al., 2011; Lidbury et al., 2014; Mausz and Chen, 2019). 
Using model marine roseobacters, we have previously studied membrane lipids in Ruegeria pomeroyi DSS-3 and Phaeobacter sp. MED193 (Sebastián et al., 2016; Smith et al., 2019). In addition to glycerophospholipids such as phosphatidylglycerol (PG) and phosphatidylethanolamine (PE), these marine roseobacters have several lipids which are not widely reported, including amino-acid containing lipids like glutamine lipid (Smith et al., 2019). Many of these roseobacters are capable of remodeling their membrane lipid composition in response to environmental change, such as adaptation to $\mathrm{P}$ stress through a phospholipase (PlcP)-mediated lipid renovation pathway by substituting glycerophospholipids with non-phosphorus containing surrogate lipids (Sebastián et al., 2016). Indeed, some roseobacter clade bacteria, including Phaeobacter sp. MED193 can produce betaine-containing membrane lipids to replace glycerophospholipids in response to phosphorus limitation (Sebastián et al., 2016).

In contrast to the aforementioned strains, some marine roseobacters are truly pelagic with small genomes, which are known to be numerically abundant in marine surface waters, along with several other bacterial groups, notably the SAR11 clade (Giebel et al., 2011; Luo and Moran, 2014, 2015; Giovannoni, 2017; Sun et al., 2017). One of such cosmopolitan pelagic roseobacter groups is the RCA (roseobacter clade affiliated cluster) group which is particularly prevalent in the bacterioplankton in temperate and polar regions of the oceans, where they can reach up to $35 \%$ of total bacterial counts (Selje et al., 2004; Giebel et al., 2009, 2011; Billerbeck et al., 2016; Zhang et al., 2016). RCA group bacteria were first isolated from the North Sea represented by the type strain Planktomarina temperata RCA23 (Giebel et al., 2013). Other RCA isolates include Roseobacter sp. LE17, isolated from an algal culture off the coast of California (Mayali et al., 2008), three strains (IMCC1909, IMCC1923, and IMCC1933) isolated from the Yellow Sea (Giebel et al., 2013) and, more recently, three RCA strains obtained using high-throughput dilution-to-extinction culturing method using seawater from the East China Sea (strains FZCC0023, FZCC0040, and FZCC0043) (Zhang et al., 2019). The genome sequence of strains RCA23 and LE17 has been reported and, interestingly, neither genome contains the genes involved in PlcP-mediated lipid remodeling, which are prevalent in other marine roseobacters (Sebastián et al., 2016). Furthermore, three single-cell amplified genomes (SAGs) belonging to the RCA were recently reported, none of which encodes the PlcP enzyme (Sun et al., 2017). This prompted us to characterize the intact membrane lipids in this ecologically important group of pelagic marine bacteria.

\section{RESULTS AND DISCUSSION}

We brought together six RCA strains isolated from geographically distinct locations around the world (Figure 1). Due to their unique growth requirements it was not feasible to use the same growth medium to cultivate all six strains. Indeed, genome sequence and comparative genomics studies have previously shown that each RCA strain has a unique requirement for vitamins (Voget et al., 2015; Zhang et al., 2019). Thus, strains RCA23, LE17, and IMCC1933 were cultivated in a diluted marine broth medium $(40 \% \mathrm{w} / \mathrm{v}$ marine broth in seawater), whereas the strains isolated from the East China Sea (strains FZCC0023, FZCC0040, and FZCC0043) do not grow in marine broth or diluted marine broth medium. Instead, they were grown in autoclaved natural seawater amended with mixed vitamins, ammonium $(1 \mathrm{mM})$, phosphate $(100 \mu \mathrm{M})$, iron, and a mixed carbon source (Zhang et al., 2019). To determine the impact of $P$ depletion on the lipid composition in these latter three RCA strains, they were also grown on the same natural seawater amended medium without adding additional phosphate. The final cell density of these six RCA strains before harvest is shown in Figure 1B. Without phosphate, the final cell density only reached $\sim 10^{7}$ cells $/ \mathrm{ml}$ whereas with phosphate amended, they reached $\sim 10^{8}$ cells $/ \mathrm{ml}$ or more (Figure 1B), suggesting $\mathrm{P}$ can be a limiting factor for their growth.

The intact membrane lipids were extracted from these six bacterial cultures using a modified Folch method and their lipid composition analyzed by liquid chromatography coupled with mass spectrometry (LC-MS) in both positive (+ve) and negative $(-\mathrm{ve})$ ionization mode (Smith et al., 2019). These lipids were separated using a hydrophilic interaction column prior to MS fragmentation and subsequent identification and quantification. Overall, four major phospholipids [phosphatidic acid (PA), PG, PE, and acyl-PG (APG)] and one aminolipid (ornithinecontaining) were consistently identified in these RCA bacterial isolates (Table 1). A representative LC-MS chromatograph of lipids from the RCA type strain (RCA23) is presented in Figure 2. The major fatty acids in these glycerophospholipids are C16:1, C18:1, and C19:1 (Supplementary Table 1) although their composition varies depending on the individual lipids (top panel). This is consistent with previous estimations of fatty acid methyl esters by gas chromatography, showing that the two monounsaturated C16:1 and C18:1 species accounted for more than $70 \%$ of the total fatty acids in strain RCA23 (Giebel et al., 2013).

In addition to PG, PE, and a small amount of PA, we also observed phospholipids that eluted at $3.9 \mathrm{~min}$ with a $\mathrm{m} / \mathrm{z}$ ranging from 925.8-967.8 (Table 1 and Figure 2). To elucidate the identity of these lipids, we isolated the major ion species with a $\mathrm{m} / \mathrm{z}$ of 925.8 for further fragmentation analysis in the negative (-ve) ionization model (Figure 3). The MS ${ }^{\mathrm{n}}$ fragmentation pattern suggests that this lipid is a modified C34:2 PG (C16:1/C18:1) by acylation (APG) with a third acyl fatty acid R3' of C12:1. Interestingly, 12-carbon fatty acids were previously found in RCA23 using gas chromatography analysis of fatty acid methyl esters (Giebel et al., 2013). APG lipids are rarely reported as a major lipid in bacteria (Yague et al., 1997; Luo et al., 2018; Hines and Xu, 2019). Indeed, APG lipids were not detected in our previous studies of the lipidomes of marine roseobacters, including Ruegeria pomeroyi DSS-3 and Phaeobacter sp. MED193 (Sebastián et al., 2016; Smith et al., 2019) nor in pelagic SAR11 clade bacteria (Carini et al., 2015). APG is produced as a minor lipid in E. coli through direct acylation of the headgroup of PG using an acyl donor, with 


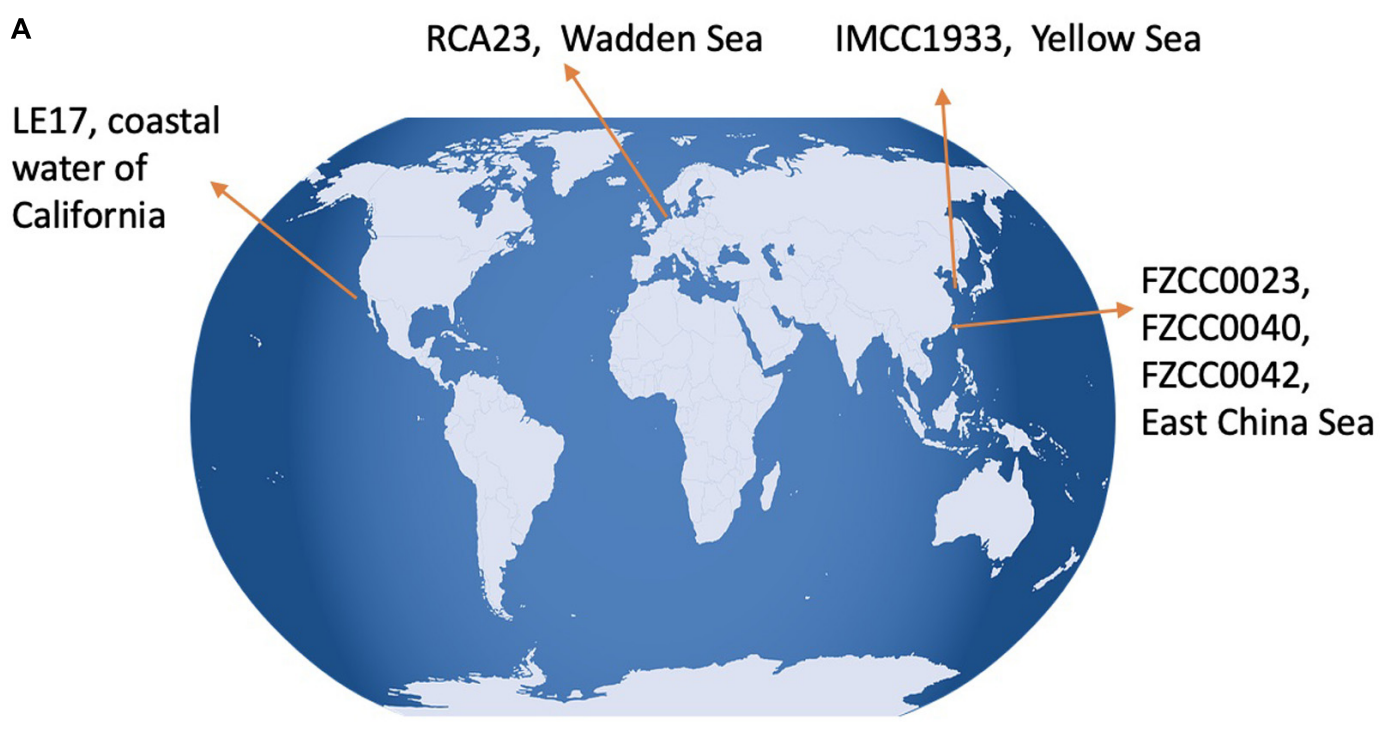

\begin{tabular}{lll}
\hline B & Medrains & Final cell density $\left(\mathbf{m l}^{-1}\right)$ \\
\hline RCA23 & $40 \%$ marine broth & $6 \times 10^{9}$ \\
IMCC1933 & $40 \%$ marine broth & $4 \times 10^{9}$ \\
LE17 & $40 \%$ marine broth & $5 \times 10^{8}$ \\
FZCC0023 & Natural seawater & $3 \times 10^{7}$ \\
& Natural seawater + phosphate & $1 \times 10^{8}$ \\
FZCC0040 & Natural seawater & $2 \times 10^{7}$ \\
& Natural seawater + phosphate & $7 \times 10^{8}$ \\
FZCC0042 & Natural seawater & $4 \times 10^{7}$ \\
& Natural seawater + phosphate & $7 \times 10^{8}$ \\
\hline
\end{tabular}

FIGURE 1|RCA isolates used in this study (A) Geographical location of the six RCA strains; (B) Culture medium used for each strain and the final cell density before harvesting.

the outer membrane lipase $\mathrm{PldB}$ involved in generating this donor (Nishijima et al., 1975; Hines and Xu, 2019). Indeed, the genome sequences of RCA isolates RCA23 and LE17 also contain a PldB homolog showing $30 \%$ sequence identity $\left(\mathrm{e}^{-20}\right)$ to that of E. coli, suggesting that APG in RCA strains is synthesized in a similar manner. However, it should be noted that the role of PldB is not limited to APG biosynthesis. Indeed, PldB of Sinorhizobium meliloti (SMc04041) also has thioesterase activity which is able to hydrolyze palmitoyl-CoA (Sahonero-Canavesi et al., 2015). Clearly the pathway for APG biosynthesis in RCA bacteria warrants further investigation.

In addition to these phospholipids, these RCA isolates also produced aminolipids. Thus, ornithine lipid (OL) eluted at $13 \mathrm{~min}$, a feature consistent with the production of OLs in R. pomeroyi DSS-3 and Phaeobacter sp. MED193 (Smith et al., 2019). OLs in these RCA isolates comprise two major species of $\mathrm{m} / \mathrm{z} 703.5$ and 717.5 respectively, and their fragmentation pattern is consistent with a fatty acid composition of 3-hydroxyl20:1/C18:1 and 3-hydroxyl20:1/C19:1, respectively. Like $R$. pomeroyi DSS-3, the genomes of RCA isolates RCA23 and LE17, and SAGs AB-661-I11 and AB-661-L17, all contain a two gene cluster ols $A$ and $o l s B$ encoding the $O$-acetyltransferase and $N$-acetyltransferase, respectively, which is likely responsible for the synthesis of OL. Glutamine lipid, which was observed in both $R$. pomeroyi DSS-3 and Phaeobacter sp. MED193, was not found in these RCA bacteria despite the fact that the $g l s B$ gene, which was previously shown to be essential for glutamine lipid biosynthesis in $R$. pomeroyi DSS-3, is present in their genomes (Smith et al., 2019).

To gain an insight into the role of $\mathrm{P}$ availability in modifying the lipidome of RCA bacteria, we used the three strains isolated from the East China Sea (strains FZCC0023, FZCC0040, and 
TABLE 1 | Major lipids identified from RCA bacteria.

\begin{tabular}{|c|c|c|c|c|}
\hline Retention time & Lipid class & $\mathrm{m} / \mathbf{z}$ & Identity & Fatty acid species \\
\hline \multirow[t]{3}{*}{$3.6 \mathrm{~min}$} & Acyl-PG (APG) & 925.8 & $(\mathrm{M}-\mathrm{H})^{-}$ & C18:1/C16:1/C12:1 \\
\hline & & 953.8 & $(\mathrm{M}-\mathrm{H})^{-}$ & C18:1/C18:1/C12:1 \\
\hline & & 967.8 & $(\mathrm{M}-\mathrm{H})^{-}$ & C18:1/C19:1/C12:1 \\
\hline \multirow[t]{3}{*}{$6.5 \min$} & $P G$ & 745.7 & $(\mathrm{M}-\mathrm{H})^{-}$ & C16:1/C18:1 \\
\hline & & 773.7 & $(\mathrm{M}-\mathrm{H})^{-}$ & C18:1/C18:1 \\
\hline & & 787.7 & $(\mathrm{M}-\mathrm{H})^{-}$ & C19:1/C18:1 \\
\hline \multirow[t]{3}{*}{$11.3 \mathrm{~min}$} & PA & 729.7 & $(\mathrm{M}+\text { acetate })^{-}$ & C16:1/C18:1 \\
\hline & & 757.7 & $(\mathrm{M}+\text { acetate })^{-}$ & C18:1/C18:1 \\
\hline & & 771.7 & $(\mathrm{M}+\text { acetate })^{-}$ & C19:1/C18:1 \\
\hline \multirow[t]{3}{*}{$11.8 \mathrm{~min}$} & $\mathrm{PE}$ & 714.7 & $(\mathrm{M}-\mathrm{H})^{-}$ & C16:1/C18:1 \\
\hline & & 742.7 & $(\mathrm{M}-\mathrm{H})^{-}$ & C18:1/C18:1 \\
\hline & & 756.7 & $(\mathrm{M}-\mathrm{H})^{-}$ & C19:1/C18:1 \\
\hline \multirow[t]{2}{*}{$13.0 \mathrm{~min}$} & $\mathrm{OL}$ & 703.7 & $(\mathrm{M}-\mathrm{H})^{-}$ & OL3-OH20:1/C18:1 \\
\hline & & 717.7 & $(\mathrm{M}-\mathrm{H})^{-}$ & OL3-OH20:1/C19:1 \\
\hline
\end{tabular}

PG, phosphatidylglycerol; APG, acyl-phosphatidylglycerol; PA, phosphatidic acid; $P E$, phosphatidylethanolamine; OL, ornithine lipid.

FZCC0043) and grew them in autoclaved natural seawater amended with $100 \mu \mathrm{M}$ phosphate or without phosphate, as described previously (see Zhang et al., 2019). Analysis of the lipidome revealed no statistical difference (student $t$-test, $p=0.31$ ) in the relative abundance of the APG lipids. However, OLs were significantly more abundant (student $t$-test, $p=0.01$ ) in the P-stressed cultures, suggesting a role of OLs in response to $\mathrm{P}$ stress (Figure 4). It should be noted that, due to the lack of commercial standards of $\mathrm{OL}$, only the relative abundance of OL/PG is analyzed in this study, which does not take into account the potential differences in ionization efficiency amongst different lipid classes. Nevertheless, a similar response of upregulation of OLs in response to $\mathrm{P}$ stress was also observed in the marine heterotroph SAR11 strain HTCC7211 (Carini et al., 2015; Sebastián et al., 2016). In SAR11 clade bacteria, lipid renovation in response to $\mathrm{P}$ stress also involves the formation of several glycolipids, which are synthesized to replace glycerophospholipids. A bifunctional glycosyltransferase (Agt) is responsible for the formation of monoglycosyldiacylglycerol (MGDG) and glucuronic acid diacylglycerol (GADG). However, no homologs of Agt were present in the genomes of RCA clade bacteria and these glycolipids were also absent in their lipidomes in our analysis.

Together, the data presented in this study reports the lipidome of six strains of RCA bacteria isolated from widely different geographical locations. These bacteria produce a relatively uncommon phospholipid, APG, although this lipid does not appear to play a role in the response to $\mathrm{P}$ stress. On the other hand, all RCA bacteria produce an ornithine-containing aminolipid which was significantly up-regulated under P stress. How these OLs facilitate membrane homeostasis in response to abiotic and biotic stresses certainly warrants further investigation.

\section{MATERIALS AND METHODS}

\section{Bacterial Strains and Cultivation}

Strains RCA23 (North Sea), IMCC1933 (Yellow Sea) and LE17 (Pacific, West coast) were grown in $50 \mathrm{ml} 40 \%(\mathrm{w} / \mathrm{v})$ marine broth medium at $15^{\circ} \mathrm{C}$ in light-dark cycles for 20 days as described by

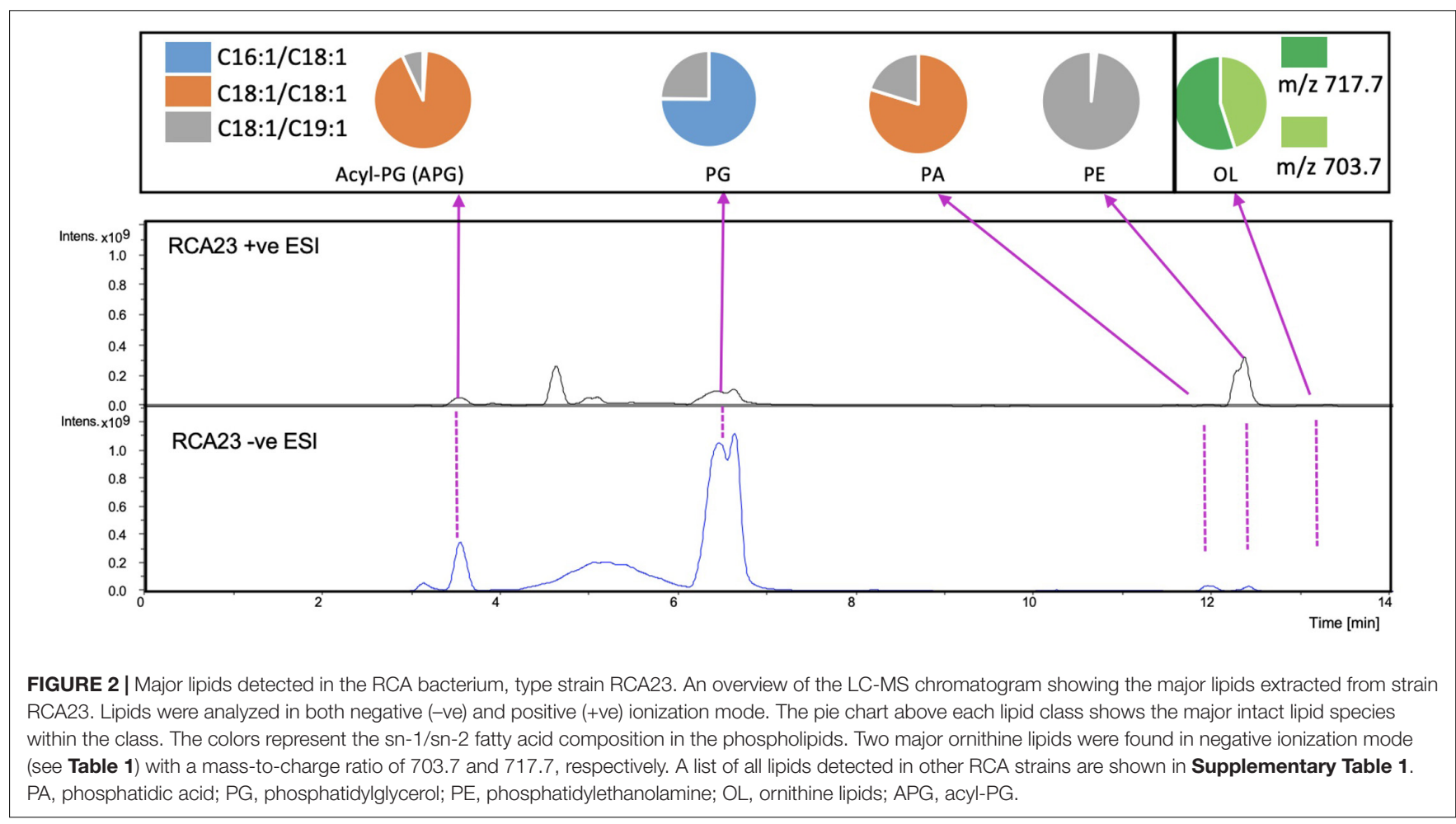




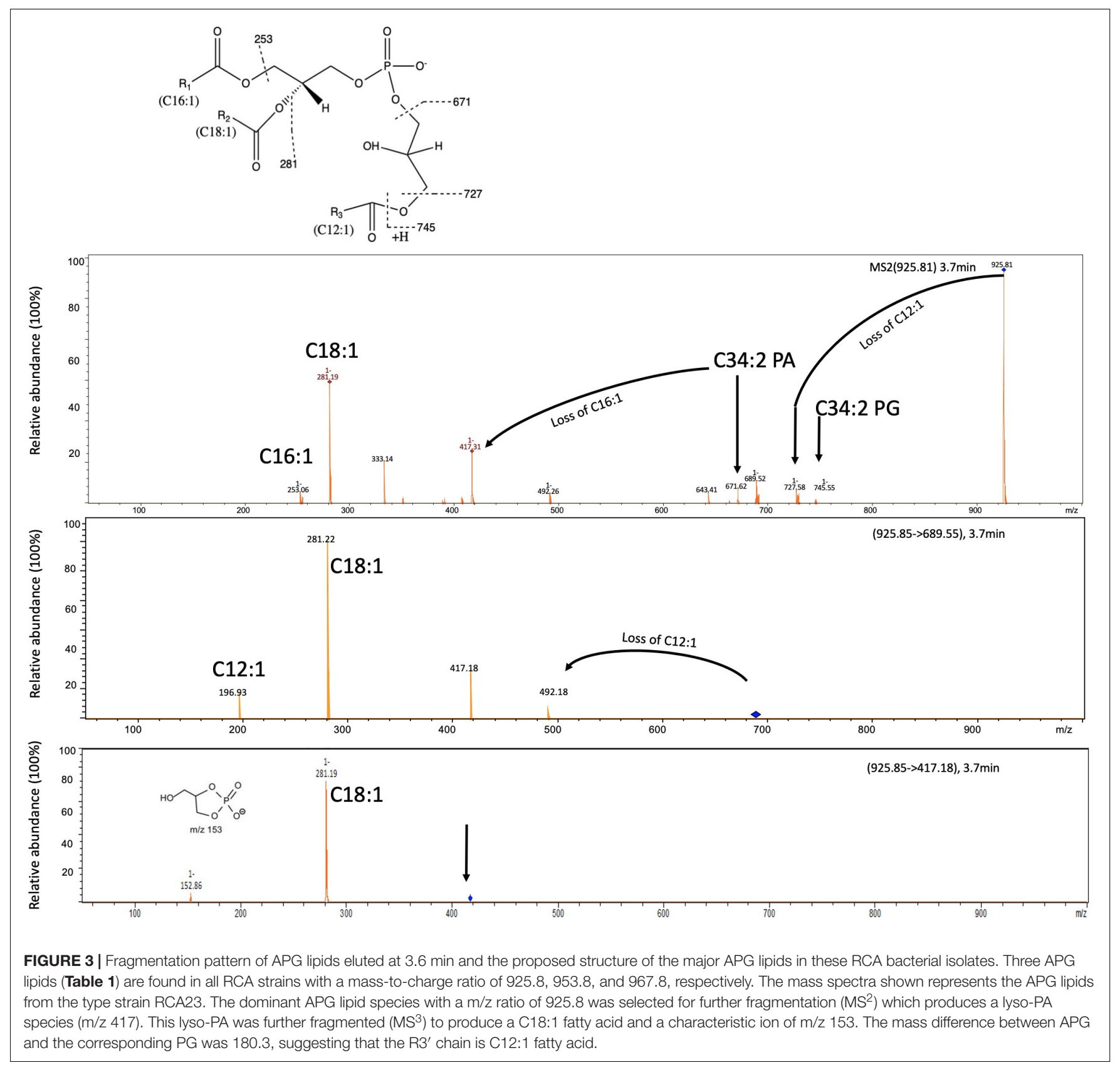

Giebel et al. (2013). End point cell density was obtained by using a BD Accuri C6 flow cytometer (BD Biosciences, San Jose, CA, United States) after the protocol of Giebel et al. (2019). Cell pellets were harvested from a $50 \mathrm{ml}$ culture by centrifugation at $4^{\circ} \mathrm{C}$. Cells were stored at $-80^{\circ} \mathrm{C}$ prior to lipid extraction.

Strains FZCC0023, FZCC0040, and FZCC0043 were isolated from the East China Sea and do not grow in marine broth or diluted marine broth medium. The seawater-based medium used for culturing these strains was prepared as follows. Coastal seawater was filtered using a $0.2-\mu \mathrm{m}$ pore-size filter and autoclaved for $90 \mathrm{~min}$. After autoclaving, seawater was sparged with $0.1 \mu \mathrm{m}$-filtered $\mathrm{CO}_{2}$ for $6 \mathrm{~h}$ followed by aeration overnight. After autoclaving and sparging, the seawater was amended with $1 \mathrm{mM} \mathrm{NH} \mathrm{NH}_{4} \mathrm{Cl}, 1 \mu \mathrm{M} \mathrm{FeCl}$, mixed carbon sources (Cho and Giovannoni, 2004) and vitamins (Carini et al., 2013). Cultures were grown in one of the following conditions: (i) phosphate-addition (100 $\mu \mathrm{M} \mathrm{K}_{2} \mathrm{HPO}_{4}$ added); or (ii) without additional phosphate (no phosphate added) (Zhang et al., 2019). Cultures were grown in the dark at $23^{\circ} \mathrm{C}$ without shaking. The cells were not washed after pre-culture; instead, the bacteria were inoculated into the same medium $(0.2 \% \mathrm{v} / \mathrm{v})$ and the cell density was monitored using a Guava EasyCyte flow cytometer before harvesting (Merck Millipore, Billerica, MA, United States). Cell pellets were harvested from $10 \mathrm{ml}$ culture by centrifugation which were stored at $-80^{\circ} \mathrm{C}$ prior to lipid extraction. 

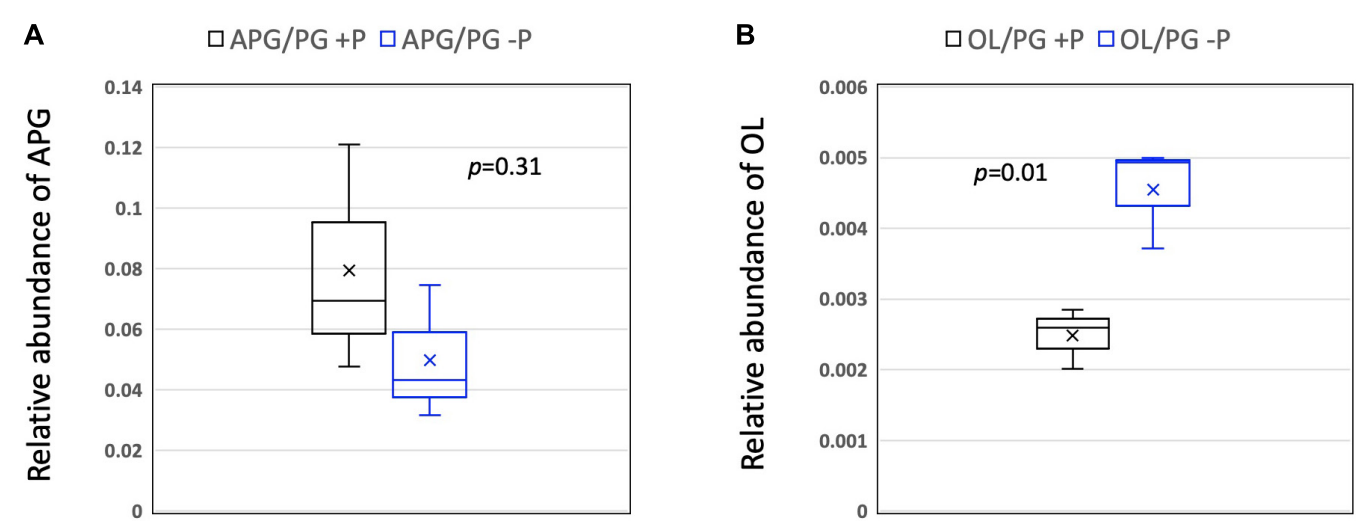

FIGURE 4 | Box-whisker plot of the relative abundance of (A) APG and (B) OL lipids (relative to PG) in medium with and without phosphorus supplementation. The line in the middle of the box represents the mean whereas the symbol $\times$ represents the median. Data were from the three isolates $(n=3)$ obtained from the East China Sea (strains FZCC0023, FZCC0040, and FZCC0043). Student-t tests were performed in Excel. PG, phosphatidylglycerol; OL, ornithine lipids; APG, acyl-PG.

\section{Lipid Extraction and HPLC-MS}

Lipid extraction from $10 \mathrm{ml}$ bacterial culture was carried out using a modified Folch extraction method (Folch et al., 1957) using HPLC-grade chloroform (1 ml), Milli-Q water $(0.3 \mathrm{ml})$ and LC-MS grade methanol $(0.5 \mathrm{ml})$ in a $2 \mathrm{ml}$ glass Chromacol vial (Smith et al., 2019). After phase separation by centrifugation, the lower chloroform phase containing the lipids was dried under nitrogen before resuspending in $0.5-1 \mathrm{~mL}$ of solvent $(0.05 \mathrm{ml}$ of $10 \mathrm{mM}$ ammonium acetate in water, $\mathrm{pH} 9.2$ and $0.95 \mathrm{ml}$ acetonitrile). The lipid d17:1/12:0 sphingosylphosphoethanolamine (Sigma-Aldrich, $50 \mathrm{nM}$ ) was added to the samples and used as internal standard. Five $\mu \mathrm{l}$ of the lipid extract was injected onto the LC-MS and separated by a Dionex 3400RS HPLC using a hydrophilic interaction column (XBridge BEH amide XP column $2.5 \mu \mathrm{m}$ $3.0 \times 150 \mathrm{~mm}$, Waters) according to their polar headgroup. Samples were run on a 15 min gradient from $95 \%(\mathrm{v} / \mathrm{v})$ acetonitrile/5\% (w/v) ammonium acetate (in water, $10 \mathrm{mM}, \mathrm{pH}$ 9.2) to $70 \%(\mathrm{v} / \mathrm{v})$ acetonitrile $/ 30 \%(\mathrm{w} / \mathrm{v})$ ammonium acetate (in water, $10 \mathrm{mM}, \mathrm{pH} 9.2$ ), followed by $5 \mathrm{~min}$ of isocratic run $70 \%$ acetonitrile/30\% ammonium acetate. Ten minutes equilibration at the initial run condition were performed between samples. The flow rate was maintained at $150 \mu \mathrm{L}$ $\mathrm{min}^{-1}$ and the column temperature at $30^{\circ} \mathrm{C}$. The injection volume was $5 \mu \mathrm{L}$ for each run; the ionization was done in both positive and negative mode. Drying conditions were the same for both modes $\left(8 \mathrm{~L} \mathrm{~min}^{-1}\right.$ drying gas at $300^{\circ} \mathrm{C}$; nebulizing gas pressure of $15 \mathrm{psi}$ ), while the end cap voltage was $4,500 \mathrm{~V}$ in positive mode and $3,500 \mathrm{~V}$ in negative mode, both with $500 \mathrm{~V}$ offset. Identification of lipid classes was made through $\mathrm{MS}^{\mathrm{n}}$ fragmentation using the amaZon SL ion trap mass spectrometer. Data analyses were carried out using the Bruker Compass Software with DataAnalysis for peak identification and lipid $\mathrm{MS}^{\mathrm{n}}$ fragmentation and QuantAnalysis for lipid quantification against internal standard sphingosylphosphoethanolamine (SPE). The abundance of each lipid was normalized against sphingosylphosphoethanolamine and expressed as relative abundance against PG.

\section{Bioinformatics and Statistics}

The genome sequences of strains RCA23 and LE17 and the singlecell amplified genomes (SAGs) of AB-661-I11, AB-661-L17, and AB-661-M21 were analyzed using the JGI IMG portal ${ }^{1}$. Genomes were searched for the presence of the following genes involved in lipid renovation: plcP (MED193_17359), olsA (SPO1979), glsB (SPO2489), and olsB (SPO1980). Student- $t$ tests were performed using Excel version 2017.

\section{DATA AVAILABILITY STATEMENT}

The original contributions presented in the study are included in the article/Supplementary Material, further inquiries can be directed to the corresponding author/s.

\section{AUTHOR CONTRIBUTIONS}

All authors listed have made a substantial, direct and intellectual contribution to the work, and approved it for publication.

\section{FUNDING}

This project has received funding from the European Research Council (ERC) under the European Union's Horizon 2020 research and innovation program (grant agreement no. 726116) and Deutsche Forschungsgemeinschaft within the Collaborative Research Centre Roseobacter (TRR51) and the Natural Environment Research Council, United Kingdom (NE/M002233/1).

\section{SUPPLEMENTARY MATERIAL}

The Supplementary Material for this article can be found online at: https://www.frontiersin.org/articles/10.3389/fmicb.2020. 552135/full\#supplementary-material

\footnotetext{
${ }^{1}$ https://img.jgi.doe.gov/
} 


\section{REFERENCES}

Billerbeck, S., Wemhauer, B., Voget, S., Poehlein, A., Giebel, H.-A., Brinkhoff, T., et al. (2016). Biogeography and environmental genomics of the Roseobacteraffiliated pelagic CHAB-I-5 lineage. Nat. Microbiol. 1:16063.

Brinkhoff, T., Giebel, H.-A., and Simon, M. (2008). Diversity, ecology, and genomics of the Roseobacter clade: a short overview. Arch. Microbiol. 189, 531-539. doi: 10.1007/s00203-008-0353-y

Buchan, A., LeCleir, G. R., Gulvik, C. A., and González, J. M. (2014). Master recyclers: features and functions of bacteria associated with phytoplankton blooms. Nat. Rev. Microbiol. 12, 686-698. doi: 10.1038/nrmicro3326

Carini, P., Steindler, L., Beszteri, S., and Giovannoni, S. J. (2013). Nutrient requirements for growth of the extreme oligotroph 'Candidatus Pelagibacter ubique' HTCC1062 on a defined medium. ISME J. 7, 592-602. doi: 10.1038/ ismej.2012.122

Carini, P., Van Mooy, B. A., Thrash, J. C., White, A., Zhao, Y., Campbell, E. O., et al. (2015). SAR11 lipid renovation in response to phosphate starvation. Proc. Natl. Acad. Sci. U S A. 112, 7767-7772. doi: 10.1073/pnas. 1505034112

Cho, J. C., and Giovannoni, S. J. (2004). Cultivation and growth characteristics of a diverse group of oligotrophic marine Gammaproteobacteria. Appl. Environ. Microbiol. 70, 432-440. doi: 10.1128/aem.70.1.432-440.2004

Curson, A. R., Todd, J. D., Sullivan, M. J., and Johnston, A. W. (2011). Catabolism of dimethylsulphoniopropionate: microorganisms, enzymes and genes. Nat. Rev. Microbiol. 9, 849-859. doi: 10.1038/nrmicro2653

Folch, J., Lees, M., and Stanley, G. H. S. (1957). A simple method for the isolation and purification of total lipids from animal tissues. J. Biol. Chem. 226, 497-509.

Giebel, H.-A., Brinkhoff, T., Zwisler, W., Selje, N., and Simon, M. (2009). Distribution of Roseobacter RCA and SAR11 lineages and distinct bacterial communities from the subtropics to the Southern Ocean. Environ. Microbiol. 11, 2164-2178. doi: 10.1111/j.1462-2920.2009.01942.x

Giebel, H.-A., Kalhoefer, D., and Lemke, A. (2011). Distribution of Roseobacter RCA and SAR11 lineages in the North Sea and characteristics of an abundant RCA isolate. ISME J. 5, 8-19. doi: 10.1038/ismej.2010.87

Giebel, H.-A., Kalhoefer, D., Gahl-Janssen, R., Choo, Y. J., Lee, K., Cho, J. C., et al. (2013). Planktomarina temperata gen. nov., sp. nov., belonging to the globally distributed RCA cluster of the marine Roseobacter clade, isolated from the German Wadden Sea. Inter. J. Sys. Evol. Microbiol. 63, 4207-4217. doi: 10.1099/ijs.0.053249-0

Giebel, H.-A., Wolterink, M., Brinkhoff, T., and Simon, M. (2019). Complementary energy acquisition via aerobic anoxygenic photosynthesis and carbon monoxide oxidation by Planktomarina temperata of the Roseobacter group. FEMS Microbiol. Ecol. 95:fiz050. doi: 10.1093/femsec/fiz050

Giovannoni, S. J. (2017). SAR11 Bacteria: the most abundant plankton in the oceans. Ann. Rev. Mar. Sci. 9, 231-255. doi: 10.1146/annurev-marine-010814015934

Hines, K. M., and Xu, L. B. (2019). Lipidomic consequences of phospholipid synthesis defects in Escherichia coli revealed by HILIC-ion mobility-mass spectrometry. Chem. Phy. Lipids. 219, 15-22. doi: 10.1016/j.chemphyslip.2019. 01.007

Lidbury, I., Murrell, J. C., and Chen, Y. (2014). Trimethylamine N-oxide metabolism by abundant marine heterotrophic bacteria. Proc. Natl. Acad. Sci. U S A. 111, 2710-2715. doi: 10.1073/pnas.1317834111

Luo, H., and Moran, M. A. (2014). Evolutionary ecology of the marine Roseobacter clade. Microbiol. Mol. Biol. Rev. 78, 573-587. doi: 10.1128/mmbr.00020-14

Luo, H., and Moran, M. A. (2015). How do divergent ecological strategies emerge among marine bacterioplankton lineages? Trends Microbiol. 23, 577-584. doi: 10.1016/j.tim.2015.05.004
Luo, Y., Javed, A. M., Deneer, H., and Chen, X. (2018). Nutrient depletioninduced production of tri-acylated glycerolphospholipids in Acinetobacter radioresistens. Sci. Rep. 8:7470.

Mausz, M. A., and Chen, Y. (2019). Microbiology and ecology of methylated amine metabolism in marine ecosystems. Curr. Issues Mol. Biol. 33, 133-148. doi: $10.21775 /$ cimb.033.133

Mayali, X., Franks, P. J., and Azam, F. (2008). Cultivation and ecosystem role of a marine Roseobacter clade-affiliated cluster bacterium. Appl. Environ. Microbiol. 74, 2595-2603. doi: 10.1128/aem.02191-07

Nishijima, M., Sa-Eki, T., Tamori, Y., Doi, O., and Nojima, S. (1975). Synthesis of acyl phosphatidylglycerol from phosphatidylglycerol in Escherichia coli K12. Evidence for the participation of detergent-resistant phospholipase A and heat-labile membrane-bound factor(s). Biochim. Biophys. Acta. 528, 107-118. doi: 10.1016/0005-2760(78)90056-5

Sahonero-Canavesi, D. X., Sohlenkamp, C., Sandoval-Calderon, M., Lamsa, A., Pogliano, K., Lopez-Lara, I. M., et al. (2015). Fatty acid-releasing activities in Sinorhizobium meliloti include unusual diacylglycerol lipase. Environ. Microbiol. 17, 3391-3406. doi: 10.1111/1462-2920.12814

Sebastián, M., Smith, A. F., González, J. M., Fredricks, H. F., van Mooy, B., Koblížek, M., et al. (2016). Lipid remodelling is a widespread strategy in marine heterotrophic bacteria upon phosphorus deficiency. ISME J. 10, 968-978. doi: 10.1038/ismej.2015.172

Selje, N., Simon, M., and Brinkhoff, T. (2004). A newly discovered Roseobacter cluster in temperate and polar oceans. Nature 427, 445-448. doi: 10.1038/ nature 02272

Smith, A. F., Rihtman, B., Stirrup, R., Silvano, E., Mausz, M. A., Scanlan, D. J., et al. (2019). Elucidation of glutamine lipid biosynthesis in marine bacteria reveals its importance under phosphorus deplete growth in Rhodobacteraceae. ISME J. 13, 39-49. doi: 10.1038/s41396-018-0249-Z

Sun, Y., Zhang, Y., Hollibaugh, J. T., and Luo, H. (2017). Ecotype diversification of an abundant Roseobacter lineage. Environ. Microbiol. 19, 1625-1638. doi: 10.1111/1462-2920.13683

Voget, S., Wemheuer, B., Brinkhoff, T., Vollmers, J., Dietrich, S., Giebel, H.-A., et al. (2015). Adaptation of an abundant Roseobacter RCA organism to pelagic systems revealed by genomic and transcriptomic analyses. ISME J. 9, 371-384. doi: 10.1038/ismej.2014.134

Yague, G., Segovia, M., and Valero-Guillen, P. L. (1997). Acyl phosphatidylglycerol: a major phospholipid of Corynebacterium amycolatum. FEMS Microbiol. Lett. 151, 125-130. doi: 10.1016/s0378-1097(97)00137-7

Zhang, Y., Sun, Y., Jiao, N., Stepanauskas, R., and Luo, H. (2016). Ecological genomics of the uncultivated marine Roseobacter lineage CHAB-I-5. Appl. Environ. Microbiol. 82, 2100-2111. doi: 10.1128/aem.03678-15

Zhang, Z., Chen, F., Chu, X., Zhang, H., Luo, H. W., Qin, F., et al. (2019). Diverse, abundant, and novel viruses infecting the marine Roseobacter RCA lineage. mSystems 4, 494-419e.

Conflict of Interest: The authors declare that the research was conducted in the absence of any commercial or financial relationships that could be construed as a potential conflict of interest.

Copyright (c) 2020 Silvano, Yang, Wolterink, Giebel, Simon, Scanlan, Zhao and Chen. This is an open-access article distributed under the terms of the Creative Commons Attribution License (CC BY). The use, distribution or reproduction in other forums is permitted, provided the original author(s) and the copyright owner(s) are credited and that the original publication in this journal is cited, in accordance with accepted academic practice. No use, distribution or reproduction is permitted which does not comply with these terms. 